Supporting Information

for

\title{
Transparent and Low-Loss Luminescent Solar Concentrators Based on Self-Trapped Exciton Emission in Lead-Free
}

\section{Double Perovskite Nanocrystals}


Oldřich Zmeškal ${ }^{\|}$, Marco Altomare ${ }^{\$}$, Andres Osvet ${ }^{\perp}$, Radek Zbořil ${ }^{\dagger}, \#$, Patrik Schmuki ${ }^{\|}$, Christoph J. Brabec ${ }^{\perp,}$, Michal Otyepkat, and Štěpán Kment ${ }^{\dot{t}, \#, *}$

${ }^{\dagger}$ Regional Centre of Advanced Technologies and Materials, Czech Advanced Technology and Research Institute, Palacky University Olomouc, Kř́žkovského 511/8, 77900 Olomouc, Czech Republic

Department of Physical Chemistry, Faculty of Science, Palacký University Olomouc, 17. listopadu 12, 71146 Olomouc, Czech Republic

\$Department of Materials Science, WW4-LKO, University of Erlangen-Nürnberg, Martensstraße 7, 91058, Erlangen, Germany

"Faculty of Chemistry, Brno University of Technology, Purkyňova 118, 61200 Brno, Czech Republic ${ }^{\perp}$ Institute i-MEET, University of Erlangen-Nürnberg, Martensstraße 7, 91058, Erlangen, Germany

\#Nanotechnology Centre, CEET, VŠB-Technical University of Ostrava, 17. listopadu 2172/15, 70800 Ostrava-Poruba, Czech Republic

"Helmholtz Institute Erlangen-Nürnberg for Renewable Energy (HI-EerN), Immerwahrstrasse 2, 91058 Erlangen, Germany

'IT4Innovations, VSB-Technical University of Ostrava, 17. listopadu 2172/15, 70800 OstravaPoruba, Czech Republic

*Correspondence to: stepan.kment@,upol.cz (S.K.) 


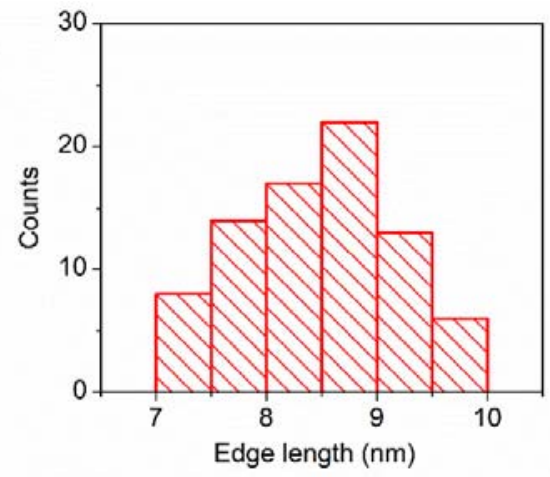

Figure S1. Edge length histogram calculated from measurements of more than 80 observed PNCs.

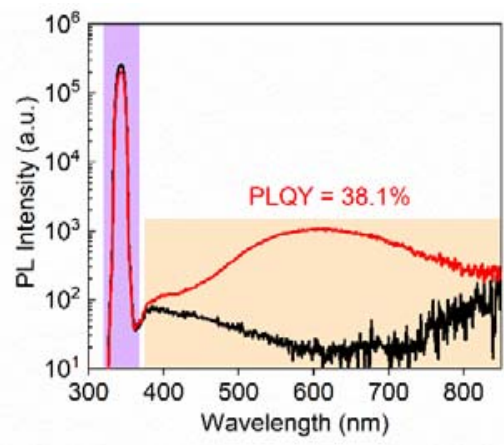

Figure S2. PL QY measurement of PNCs in colloidal suspension (excitation and PL emission regions are highlighted in purple and orange, respectively).


Figure S3. Optical properties of PNCs in PMMA matrix. (a) Excitation-emission color map. (b) Absorption (blue curve), normalized PL excitation (green curve, $\lambda_{\mathrm{em}}=605 \mathrm{~nm}$ ) and PL emission (red curve, $\lambda_{\mathrm{ex}}=343 \mathrm{~nm}$ ) spectra. (c) Time-resolved PL decay $\left(\lambda_{\mathrm{em}}=605 \mathrm{~nm}\right.$ ) with corresponding stretched-exponential fit (red line). 


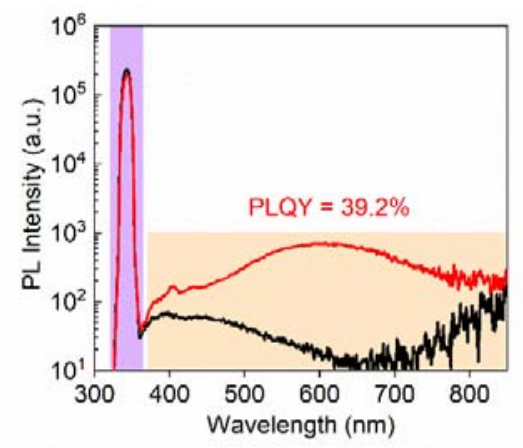

Figure S4. PL QY measurement of PNCs in PMMA (excitation and PL emission regions are highlighted in purple and orange, respectively).


Figure S5. (a) Scheme of internal OQE measurements in integrating sphere. (b) Photograph of integrating sphere used for optical measurements together with fabricated LSC of different sizes $(2 \times 2,4 \times 4,6 \times 6$ and $8 \times 8 \mathrm{~cm}^{2}$ ) with edges masked with a black nontransparent tape. 

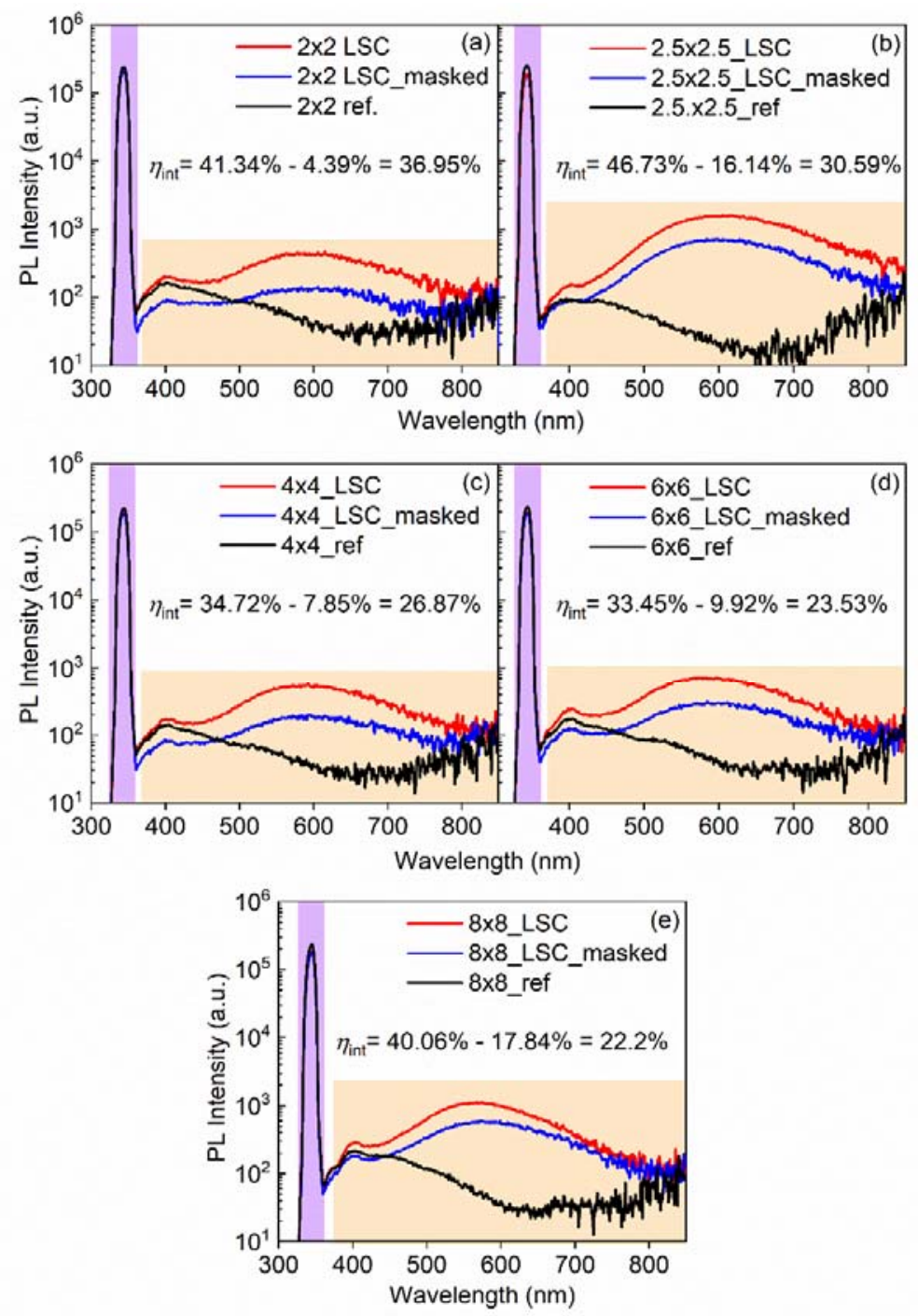

Figure S6. PL QY measurements of PNC LSCs with sizes of $2 \times 2,2.5 \times 2.5,4 \times 4,6 \times 6$ and $8 \times 8 \mathrm{~cm}^{2}$ with and without masked edges together with calculations of internal OQEs (excitation and PL emission regions are highlighted in purple and orange, respectively). 

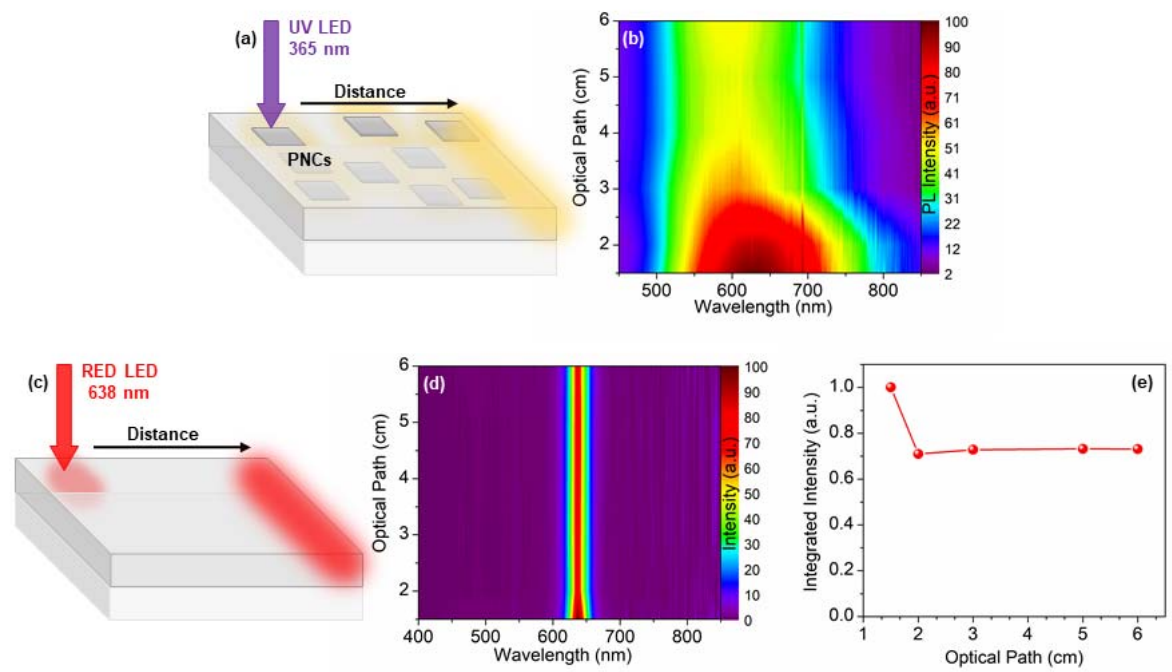

Figure S7. Emission intensity vs. optical path measurements. (a) Scheme of experimental setup (PNC LSC). (b) Color map representing PL emission vs. optical path. (c) Scheme of experimental setup (PMMA thin layer deposited on glass slab). (d) Color map representing emission collected from the edge of glass slab with PMMA thin film as a function of optical path. (e) Corresponding integrated emission intensity as a function of optical path.

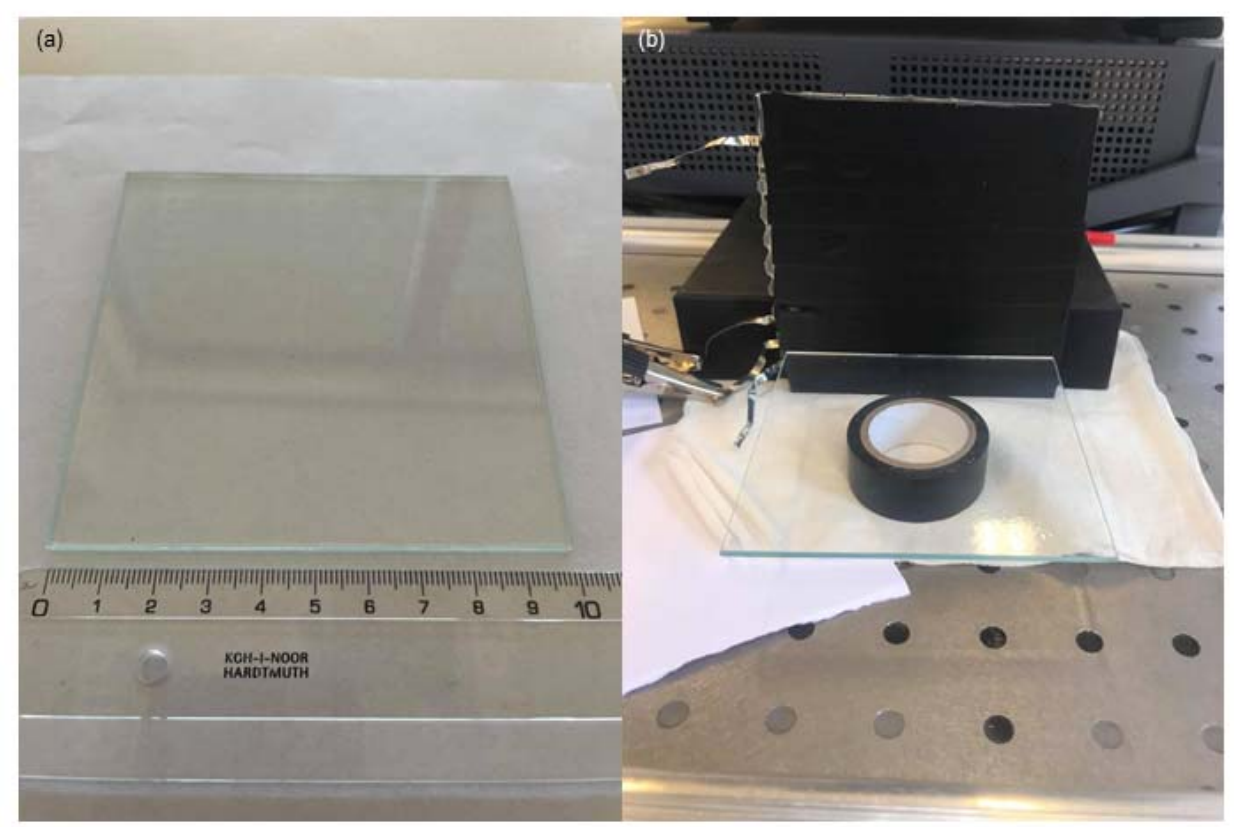

Figure S8. (a) Photograph of $10 \times 10 \mathrm{~cm}^{2}$ PNC LSC. (b) The same LSC coupled to a calibrated a-Si solar cell. 


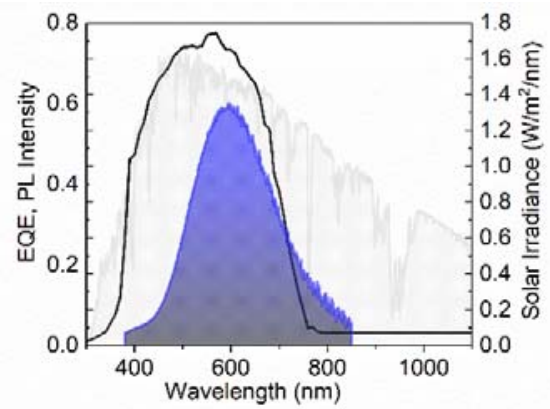

Figure S9. The EQE spectrum of a-Si solar cell compared with AM 1.5G spectrum (highlighted in grey) together with PL emission peak of PNC LSC (highlighted in blue).



Figure S10. Transmittance spectrum of $10 \times 10 \mathrm{~cm}^{2}$ PNC LSC.



Figure S11. (a) Internal OQE of PNCs-based LSCs simulated for different PL QYs and LSC lateral sizes (solid lines with symbols) and measured for PNC-based LSCs with the size from $2 \times 2 \mathrm{~cm}^{2}$ till $10 \times 10 \mathrm{~cm}^{2}$ (dots).

The difference between experimental and simulated values of internal optical quantum efficiencies (red dots and blue circles) for smaller lateral lengths of LSC in Fig. S11 can be attributed to the choice of using the same absorption and emission spectra as inputs in all the MC simulations. Moreover, the difference can be explained by the slightly smaller range of absorption and emission spectra employed in our simulations due to software requirements. Finally, bigger differences for smaller glasses were also reported in the literature. ${ }^{1}$ 
Table S1. Overview of literature results for LSCs based on semiconductor QDs, CDs and PNCs.

\begin{tabular}{|c|c|c|c|c|c|c|c|}
\hline $\begin{array}{c}\text { Type of } \\
\text { luminophore }\end{array}$ & $\begin{array}{c}\text { Dimensions } \\
(\mathbf{c m} \times \mathbf{c m} \times \mathbf{c m})\end{array}$ & Light source & $\begin{array}{c}\text { PLQY } \\
(\%)\end{array}$ & $\begin{array}{r}\eta_{\text {int }} \\
(\%)\end{array}$ & $\begin{array}{l}\eta_{\mathrm{ext}} \\
(\%)\end{array}$ & $C_{\mathrm{int}}$ & Ref. \\
\hline Si QDs & $12 \times 12 \times 0.26$ & Solar simulator & 46 & 30.0 & - & 3.5 & 1 \\
\hline Mn-doped ZnSe QDs & $2.7 \times 7.5 \times 0.042$ & $400 \mathrm{~nm}$ & 53 & 37.2 & - & - & 2 \\
\hline $\begin{array}{l}\text { CdSe/CdS core/shell } \\
\text { QDs }\end{array}$ & $2 \times 2 \times 0.2$ & $400 \mathrm{~nm}$ & 86 & 48.0 & - & 1.2 & 3 \\
\hline $\begin{array}{l}\text { CdSe/CdS core/shell } \\
\text { QDs }\end{array}$ & $21.5 \times 1.3 \times 0.5$ & Solar simulator & 45 & 10.2 & 0.6 & 2.2 & 4 \\
\hline $\begin{array}{l}\mathrm{CuInSeS}_{2} \mathrm{In} / \mathrm{ZnS} \\
\text { core/shell QDs }\end{array}$ & $12 \times 12 \times 0.3$ & Solar simulator & 40 & 16.7 & 3.3 & 1.7 & 5 \\
\hline $\begin{array}{l}\text { Silica-coated } \\
\mathrm{CdSe} / \mathrm{CdZn}_{\mathrm{x}} \mathrm{S}_{1-\mathrm{x}} \\
\text { core/alloyed shell } \\
\text { QDs }\end{array}$ & $10 \times 10 \times 0.16$ & $405 \mathrm{~nm}$ & 57 & 24.0 & - & 3.8 & 6 \\
\hline $\begin{array}{l}\text { Silica-coated } \\
\mathrm{CdSe} / \mathrm{CdZn}_{\mathrm{x}} \mathrm{S}_{1-\mathrm{x}} \\
\text { core/alloyed shell } \\
\text { QDs }\end{array}$ & $10 \times 10 \times 0.16$ & sunlight & - & 21.0 & 1.9 & 3.3 & 6 \\
\hline $\mathrm{CuInS}_{2} / \mathrm{CdS}$ QDs & $7.5 \times 7.5 \times 0.28$ & - & 75 & - & 5.7 & - & 7 \\
\hline $\begin{array}{l}\mathrm{PbS} / \mathrm{CdS} \text { core/shell } \\
\text { QDs }\end{array}$ & $10 \times 1.5 \times 0.2$ & Solar simulator & $40-50$ & 4.5 & 1.1 & 2.3 & 8 \\
\hline $\begin{array}{l}\text { CDs/PLMA } \\
\text { CDs/PVP }\end{array}$ & - & Solar simulator & 30 & 4.0 & - & 1.5 & 9 \\
\hline $\begin{array}{l}\text { CDs/PLMA } \\
\text { CDs/PVP }\end{array}$ & $2 \times(10 \times 10 \times 0.1)$ & Solar simulator & - & - & 1.1 & - & 9 \\
\hline $\mathrm{CsPb}\left(\mathrm{Br}_{\mathrm{x}} \mathrm{I}_{1-\mathrm{x}}\right)_{3} \mathrm{NCs}$ & $9.0 \times 1.3 \times 0.2$ & Solar simulator & 60 & 5.0 & 2.0 & - & 10 \\
\hline N-CDs & $2.5 \times 1.6 \times 0.1$ & Solar simulator & - & - & 4.8 & - & 11 \\
\hline $\begin{array}{l}\mathrm{Mn}^{2+} \text {-doped } \\
\text { Perovskite NCs }\end{array}$ & $20 \times 20 \times 0.5$ & $395 \mathrm{~nm}$ & 10 & 7.5 & - & 0.8 & 12 \\
\hline $\mathrm{CuInSe}_{2} / \mathrm{ZnS}$ QDs & $12 \times 12 \times 0.16$ & $385 \mathrm{~nm}$ & 72 & 19.0 & 5.1 & 3.6 & 13 \\
\hline $\begin{array}{l}\mathrm{Mn}: \mathrm{Cd}_{\mathrm{x}} \mathrm{Zn}_{1-\mathrm{x}} \mathrm{S} / \mathrm{ZnS} \\
\text { QDs }\end{array}$ & $12 \times 12 \times 0.16$ & $385 \mathrm{~nm}$ & 78 & 58.0 & 3.4 & 10.9 & 13 \\
\hline $\mathrm{CuInS}_{2} / \mathrm{ZnS}$ QDs & $10 \times 10 \times 0.14$ & Solar simulator & 91 & 23.0 & 8.1 & 4.1 & 14 \\
\hline
\end{tabular}




\begin{tabular}{|c|c|c|c|c|c|c|c|}
\hline $\begin{array}{c}\text { Type of } \\
\text { luminophore }\end{array}$ & $\begin{array}{l}\text { Dimensions } \\
(\mathrm{cm} \times \mathbf{c m} \times \mathbf{c m})\end{array}$ & Light source & $\begin{array}{l}\text { PLQY } \\
(\%)\end{array}$ & $\begin{array}{r}\eta_{\text {int }} \\
(\%)\end{array}$ & $\begin{array}{l}\eta_{\text {ext }} \\
(\%)\end{array}$ & $\boldsymbol{C}_{\text {int }}$ & Ref. \\
\hline $\begin{array}{l}\mathrm{Yb}^{3+} \text {-doped } \mathrm{CsPbCl}_{3} \\
\mathrm{NCs}\end{array}$ & $5 \times 5 \times 0.2$ & $365 \mathrm{~nm}$ & 164 & 118.0 & 3.7 & 7.4 & 15 \\
\hline $\mathrm{N}-\mathrm{CDs}$ & $2 \times 2 \times 0.2$ & Solar simulator & - & - & 12.2 & - & 16 \\
\hline CDs & $10 \times 10 \times 0.2$ & Solar simulator & 50 & - & 0.3 & - & 17 \\
\hline $\mathrm{CsPb}\left(\mathrm{Br}_{1-\mathrm{x}} \mathrm{I}_{\mathrm{x}}\right)_{3} \mathrm{NCs}$ & $10 \times 10 \times 0.2$ & Solar simulator & 70 & - & 1.1 & - & 17 \\
\hline $\mathrm{CsPb}\left(\mathrm{Cl}_{\mathrm{x}} \mathrm{Br}_{1-\mathrm{x}}\right)_{3} \mathrm{NCs}$ & $10 \times 10 \times 0.2$ & Solar simulator & 60 & - & 1.7 & - & 17 \\
\hline CNDs & $3 \times 3 \times 0.3$ & $354 \mathrm{~nm}$ & 45 & 22.0 & 12.0 & 0.6 & 18 \\
\hline CDs & $1.8 \times 1.8 \times 0.11$ & Solar simulator & 80 & - & 5.0 & - & 19 \\
\hline $\mathrm{N}-\mathrm{CDs}$ & $5.0 \times 2.5 \times 0.2$ & Solar simulator & - & - & 4.5 & - & 20 \\
\hline PNPLs & $2 \times 2 \times 0.2$ & $400 \mathrm{~nm}$ & 81 & 51.0 & - & 1.0 & 21 \\
\hline PNPLs & $10 \times 10 \times 0.2$ & sunlight & - & 26.0 & 0.9 & 3.3 & 21 \\
\hline $\mathrm{CuInS}_{2}$ QDs & $30 \times 30 \times 0.5$ & Solar simulator & 85 & 29.2 & 6.8 & 4.4 & 22 \\
\hline CDs & $10 \times 10 \times 1$ & Solar simulator & 40 & - & 0.9 & - & 23 \\
\hline $\mathrm{CdSe} / \mathrm{CdS}$ QDs & $10 \times 10 \times 0.4$ & Solar simulator & 36 & - & 3.0 & - & 24 \\
\hline CDs & $6.5 \times 2.0 \times 0.1$ & Solar simulator & 80 & - & 1.7 & - & 25 \\
\hline AIE molecules & $6.5 \times 2.0 \times 0.2$ & Solar simulator & 35 & - & 2.3 & - & 25 \\
\hline $\mathrm{Cs}_{4} \mathrm{PbBr}_{6} \mathrm{NCs}$ & $10 \times 10 \times 0.4$ & Solar simulator & 58 & 25.0 & 2.4 & 1.6 & 26 \\
\hline CDs & $5 \times 5 \times 0.3$ & Solar simulator & 46 & - & 5.3 & - & 27 \\
\hline Organic dyes & $5 \times 5 \times 0.3$ & Solar simulator & 87 & - & 13.4 & - & 27 \\
\hline CDs & $8 \times 8 \times 0.8$ & Solar simulator & $46-74$ & 23.6 & 2.3 & 0.6 & 28 \\
\hline $\begin{array}{l}\mathrm{CuInS}_{2} / \mathrm{ZnSe}_{\mathrm{x}} \mathrm{S}_{1-\mathrm{x}} \\
\text { QDs }\end{array}$ & $10 \times 10 \times 1$ & Solar simulator & 59 & - & 1.0 & - & 29 \\
\hline $\mathrm{CuInS}_{2} / \mathrm{ZnS}$ QDs & $15.24 \times 15.24 \times 1$ & RGB light & - & 16 & 10.8 & 0.6 & 30 \\
\hline $\begin{array}{l}\text { Cu-doped ZnInSe } \\
\text { QDs }\end{array}$ & $8 \times 8 \times 0.2$ & Solar simulator & 63 & - & 3.7 & - & 31 \\
\hline $\mathrm{Si} \mathrm{NCs}+\mathrm{DPA}$ & $5.4 \times 2.05 \times 0.29$ & Solar simulator & 45 & - & 4.3 & - & 23 \\
\hline PNPLs & $10 \times 10 \times 0.2$ & Solar simulator & 56 & - & 2.0 & - & 33 \\
\hline $\mathrm{CsPbI}_{3} \mathrm{NCs}$ & $2 \times 2$ & Solar simulator & 98 & 36.0 & - & - & 34 \\
\hline $\mathrm{CsPbI}_{3} \mathrm{NCs}$ & $15 \times 5$ & Solar simulator & 98 & - & 3.1 & - & 34 \\
\hline
\end{tabular}




\begin{tabular}{|l|c|c|c|c|c|c|c|}
\hline \multicolumn{1}{|c|}{$\begin{array}{c}\text { Type of } \\
\text { luminophore }\end{array}$} & $\begin{array}{c}\text { Dimensions } \\
(\mathbf{c m} \times \mathbf{c m} \times \mathbf{c m})\end{array}$ & Light source & $\begin{array}{c}\text { PLQY } \\
\mathbf{( \% )}\end{array}$ & $\begin{array}{c}\eta_{\text {int }} \\
\mathbf{( \% )}\end{array}$ & $\begin{array}{c}\eta_{\text {ext }} \\
\mathbf{( \% )}\end{array}$ & $\boldsymbol{C}_{\text {int }}$ & Ref. \\
\hline $\begin{array}{l}\text { Copper-doped } \\
\mathrm{InP} / \mathrm{ZnSe} \mathrm{QDs}\end{array}$ & $10 \times 10 \times 0.2$ & Solar simulator & 81 & 37.2 & 5.9 & 4.7 & 35 \\
\hline $\begin{array}{l}\mathrm{Mn}^{2+} / \mathrm{Yb}^{3+} \text { co-doped } \\
\mathrm{CsPbCl}\end{array}$ PNCs & $13 \times 13 \times 0.5$ & Solar simulator & 125 & $\sim 70$ & 7.3 & $\sim 4.6$ & 36 \\
\hline $\mathrm{CDs}$ & $15 \times 15 \times 0.5$ & Solar simulator & 65 & - & 2.2 & - & 37 \\
\hline $\mathrm{InP} / \mathrm{ZnO} \mathrm{QDs}$ & $9 \times 1.5 \times 0.3$ & Solar simulator & 18 & - & 1.5 & - & 38 \\
\hline $\mathrm{InP} / \mathrm{ZnS}$ & $6 \times 6 \times 2$ & Solar simulator & 36 & 22.2 & 1.7 & 1.7 & 39 \\
\hline This work & $2 \times 2 \times 0.2$ & 343 nm & 41 & 37.0 & - & 1.0 & \\
\hline This work & $10 \times 10 \times 0.2$ & Solar simulator & - & 21.2 & 0.7 & 2.7 & \\
\hline
\end{tabular}

Table S2. ICP-MS results and calculations.

\begin{tabular}{|c|c|c|c|c|c|c|c|c|}
\hline $\begin{array}{c}\text { Feeding } \\
\text { ratio of } \\
\mathrm{Na}(\mathrm{x})\end{array}$ & $\begin{array}{c}\mathrm{Na} \\
(\mathrm{mg} / \mathrm{L})\end{array}$ & $\begin{array}{c}\mathrm{In} \\
(\mathrm{mg} / \mathrm{L})\end{array}$ & $\begin{array}{c}\mathrm{Bi} \\
(\mathrm{mg} / \mathrm{L})\end{array}$ & $\begin{array}{c}\mathrm{Na} \\
(\mathrm{mmol} / \mathrm{L})\end{array}$ & $\begin{array}{c}\mathrm{In} \\
(\mathrm{mmol} / \mathrm{L})\end{array}$ & $\begin{array}{c}\mathrm{Bi} \\
(\mathrm{mmol} / \mathrm{L})\end{array}$ & $\begin{array}{c}\mathrm{Na} / \mathrm{In} \\
\mathrm{Bi} / \mathrm{In} \\
(\%)\end{array}$ \\
\hline 0.6 & 138.43 & 1001.57 & 13.08 & 6.01 & 8.7 & 0.062 & 0.69 & 0.71 \\
\hline
\end{tabular}

\section{Computational details}

Monte Carlo ray-trace simulation is based on a repeated random sampling, which is applied to determine the fate of many simulated photons within the LSC. Each simulated photon encounters many events, and their outcomes are determined using random number generation weighted by calculated probabilities based on the properties of the system. The events occurring in the MC raytracing simulation are displayed in Fig. S12a. A graphical user interface (GUI) was used for each MC ray-tracing simulation. For more details on the specific setup with the help of GUI of the code, please see Ref. 40 and Fig. S12b. 


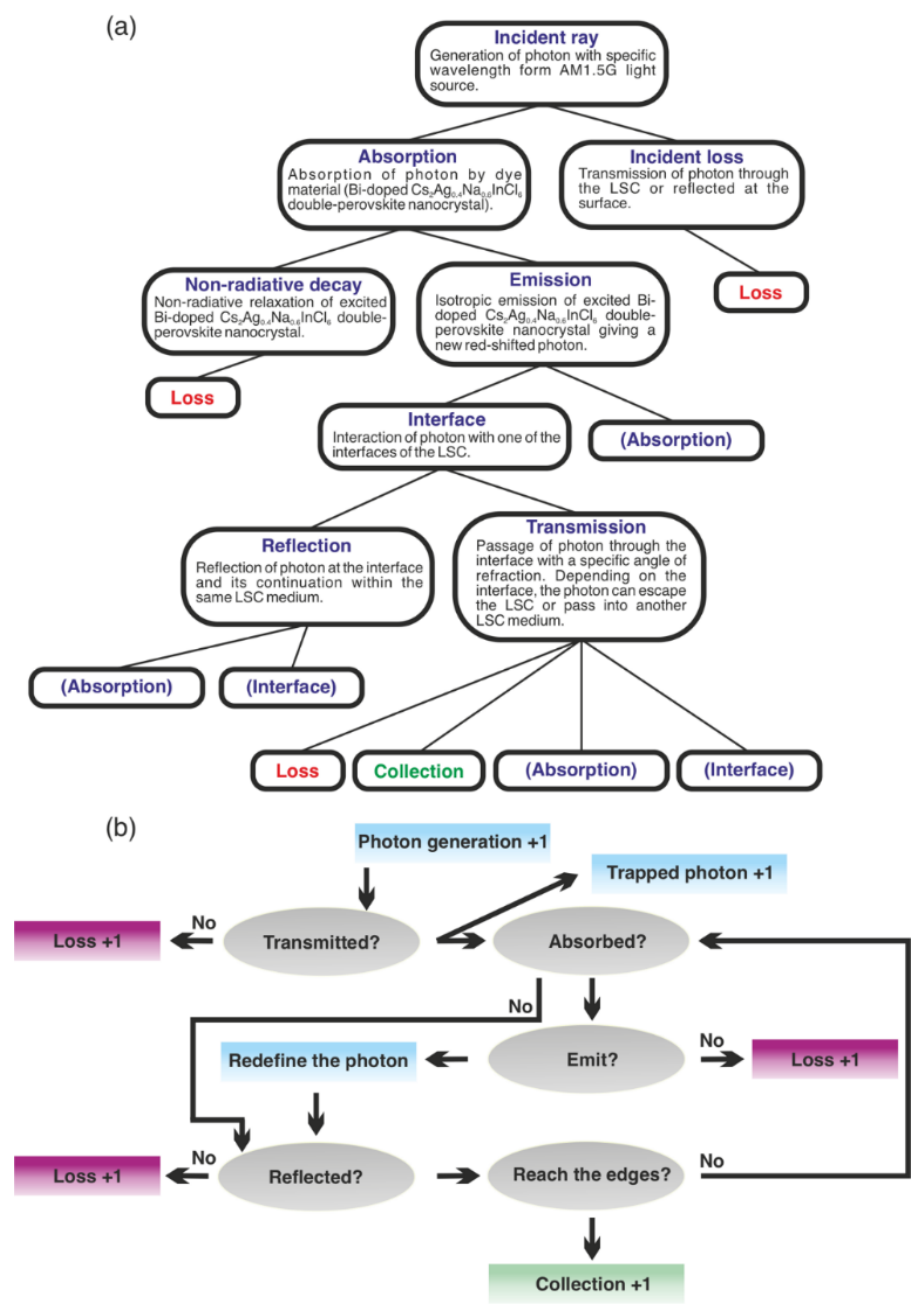

Figure S12. (a) Overview of the processes occurring during the MC simulation. (b) A flow chart showing a cycle employed in the MC simulation.

\section{References:}

[1] F. Meinardi, S. Ehrenberg, L. Dhamo, F. Carulli, M. Mauri, F. Bruni, R. Simonutti, U. Kortshagen, S. Brovelli, Nat. Photonics 2017, 11, 177.

[2] C. S. Erickson, L. R. Bradshaw, S. McDowall, J. D. Gilbertson, D. R. Gamelin, D. L. Patrick, ACS Nano 2014, 8, 3461.

[3] I. Coropceanu, M. G. Bawendi, Nano Lett. 2014, 14, 4097.

[4] F. Meinardi, A. Colombo, K. A. Velizhanin, R. Simonutti, M. Lorenzon, L. Beverina, R. Viswanatha, V. I. Klimov, S. Brovelli, Nat. Photonics 2014, 8, 392.

[5] F. Meinardi, H. McDaniel, F. Carulli, A. Colombo, K. A. Velizhanin, N. S. Makarov, R. Simonutti, V. I. Klimov, S. Brovelli, Nat. Nanotechnol 2015, 10, 878.

[6] H. Li, K. Wu, J. Lim, H.-J. Song, V. I. Klimov, Nat. Energy 2016, 1.

[7] R. Sumner, S. Eiselt, T. B. Kilburn, C. Erickson, B. Carlson, D. R. Gamelin, S. McDowall, D. L. Patrick, J. Phys. Chem. C 2017, 121, 3252.

[8] Y. Zhou, D. Benetti, Z. Fan, H. Zhao, D. Ma, A. O. Govorov, A. Vomiero, F. Rosei, Adv. Energy Mater. 2016, 6, 1501913. 
[9] Y. Zhou, D. Benetti, X. Tong, L. Jin, Z. M. Wang, D. Ma, H. Zhao, F. Rosei, Nano Energy 2018, 44, 378.

[10] H. Zhao, Y. Zhou, D. Benetti, D. Ma, F. Rosei, Nano Energy 2017, 37, 214.

[11] Y. Li, P. Miao, W. Zhou, X. Gong, X. Zhao, J. Mater. Chem. A 2017, 5, 21452.

[12] F. Meinardi, Q. A. Akkerman, F. Bruni, S. Park, M. Mauri, Z. Dang, L. Manna, S. Brovelli, ACS Energy Lett. 2017, 2, 2368.

[13] K. Wu, H. Li, V. I. Klimov, Nat. Photonics 2018, 12, 105.

[14] M. R. Bergren, N. S. Makarov, K. Ramasamy, A. Jackson, R. Guglielmetti, H. McDaniel, ACS Energy Lett. 2018, 3, 520.

[15] X. Luo, T. Ding, X. Liu, Y. Liu, K. Wu, Nano Lett. 2019, 19, 338.

[16] X. Gong, W. Ma, Y. Li, L. Zhong, W. Li, X. Zhao, Org. Electron. 2018, 63, 237.

[17] H. Zhao, D. Benetti, X. Tong, H. Zhang, Y. Zhou, G. Liu, D. Ma, S. Sun, Z. M. Wang, Y. Wang, F. Rosei, Nano Energy 2018, 50, 756.

[18] M. J. Talite, H. Y. Huang, Y. H. Wu, P. G. Sena, K. B. Cai, T. N. Lin, J. L. Shen, W. C. Chou, C. T. Yuan, ACS Appl. Mater. Interfaces 2018, 10, 34184.

[19] Z. Wang, X. Zhao, Z. Guo, P. Miao, X. Gong, Org. Electron. 2018, 62, 284.

[20] F. Mateen, M. Ali, H. Oh, S.-K. Hong, Sol. Energy 2019, 178, 48.

[21] M. Wei, F. P. G. de Arquer, G. Walters, Z. Yang, L. N. Quan, Y. Kim, R. Sabatini, R. Quintero-Bermudez, L. Gao, J. Z. Fan, F. Fan, A. Gold-Parker, M. F. Toney, E. H. Sargent, Nat. Energy 2019, 4, 197.

[22] A. Anand, M. L. Zaffalon, G. Gariano, A. Camellini, M. Gandini, R. Brescia, C. Capitani, F. Bruni, V. Pinchetti, M. Zavelani-Rossi, F. Meinardi, S. A. Crooker, S. Brovelli, Adv. Funct. Mater. 2019, 30.

[23] H. Zhao, J. Lumin. 2019, 211, 150.

[24] G. Liu, R. Mazzaro, Y. Wang, H. Zhao, A. Vomiero, Nano Energy 2019, 60, 119.

[25] W. Ma, W. Li, R. Liu, M. Cao, X. Zhao, X. Gong, Chem. Commun. 2019, 55, 7486.

[26] H. Zhao, R. Sun, Z. Wang, K. Fu, X. Hu, Y. Zhang, Adv. Funct. Mater. 2019, 29.

[27] F. Mateen, M. Ali, S. Y. Lee, S. H. Jeong, M. J. Ko, S.-K. Hong, Sol. Energy 2019, 190, 488.

[28] L. Zdražil, S. Kalytchuk, K. Hola, M. Petr, O. Zmeskal, S. Kment, A. L. Rogach, R. Zboril, Nanoscale 2020, 12, 6664.

[29] D. C. J. Neo, W. P. Goh, H. H. Lau, J. Shanmugam, Y. F. Chen, ACS Appl. Nano Mater. 2020, 3, 6489 .

[30] A. R. M. Velarde, E. R. Bartlett, N. S. Makarov, C. Castañeda, A. Jackson, K. Ramasamy, M. R. Bergren, H. McDaniel, ACS Appl. Energy Mater. 2020, 3, 9, 8159-8163.

[31] X. Liu, B. Luo, J. Liu, D. Jing, D. Benetti, F. Rosei, J. Mater. Chem. A 2020, 8, 1787.

[32] R. Mazzaro, A. Gradone, S. Angeloni, G. Morselli, P. G. Cozzi, F. Romano, A. Vomiero, P. Ceroni, ACS Photonics 2019, 6, 2303.

[33] Z. Li, A. Johnston, M. Wei, M. I. Saidaminov, J. Martins de Pina, X. Zheng, J. Liu, Y. Liu, O. M. Bakr, E. H. Sargent, Joule 2020, 4, 631.

[34] J. Wu, J. Tong, Y. Gao, A. Wang, T. Zhang, H. Tan, S. Nie, Z. Deng, Angew. Chem., Int. Ed. 2020, 59, 7738.

[35] S. Sadeghi, H. Bahmani Jalali, S. B. Srivastava, R. Melikov, I. Baylam, A. Sennaroglu, S. Nizamoglu, iScience 2020, 23, 101272.

[36] T. Cai, J. Wang, W. Li, K. Hills-Kimball, H. Yang, Y. Nagaoka, Y. Yuan, R. Zia, O. Chen, Adv. Sci. 2020, 7, 2001317.

[37] H. Zhao, G. Liu, S. You, F. V. A. Camargo, M. Zavelani-Rossi, X. Wang, C. Sun, B. Liu, Y. Zhang, G. Han, A. Vomiero, X. Gong, Energy Environ. Sci. 2021, 14, 396-406.

[38] S. Sadeghi, H. Bahmani Jalali, R. Melikov, B. Ganesh Kumar, M. Mohammadi Aria, C. W. Ow-Yang, S. Nizamoglu, ACS Appl. Mater. Interfaces 2018, 10, 12975. 
[39] H. Bahmani Jalali, S. Sadeghi, I. Baylam, M. Han, C. W. Ow-Yang, A. Sennaroglu, S. Nizamoglu, Nano Research 2020, 14, 1488.

[40] B. Zhang, H. Yang, T. Warner, P. Mulvaney, G. Rosengarten, W. W. H. Wong, K. P. Ghiggino, Methods Appl. Fluoresc. 2020, 8, 037001. 